\title{
Endoscopic ultrasound-guided fine-needle aspiration for the diagnosis of retroperitoneal schwannoma
}

Schwannoma is a rare peripheral nerve sheath tumor that is difficult to diagnose by imaging features alone. Endoscopic ultrasound (EUS)-guided fine-needle aspiration (FNA) with specific immunohistochemical (IHC) staining may be the only tool to obtain a diagnostic sample from such lesions. There are only a few case reports describing EUS-FNA diagnosis of retroperitoneal Schwannoma [1,2]. In this report, we describe four cases (three males; mean age: $54.5 \pm 16.4$ years) with retroperitoneal Schwannoma, in whom the diagnosis was achieved with EUS-FNA and adjunctive IHC staining.

All lesions were well demarcated, and had a rounded contour ( $\checkmark$ Fig. 1 a).

The mean size of the lesions on EUS was $23.7 \pm 3.6 \mathrm{~mm}$. EUS-FNA was successfully performed with a 22-gauge needle in all cases ( Fig. 1 b), with a sufficient yield for both cytological and cellblock analysis. The median number of needle passes was 2.5 (range $2-3$ ). The cellblock analysis revealed bland proliferation of spindle cells with a palisading appearance and wavy fibrillar architecture ( Fig. 1 c). Further evaluation with IHC revealed negative staining for actin, CD34, CD-117, and strong positive staining for S-100 antibody in all cases ( Fig. 1 d). Further evaluation of the cellular proliferative activity was studied with Ki-67 staining, and a low proliferation rate (Ki-67 < 5\%) was reported in all cases, supporting the benign nature of the lesions.

We recommended conservative follow-up for our patients rather than surgical resection, because all of the patients were asymptomatic and there were no mitotic figures on FNA, with a low Ki-67 index in all the aspirates. It is worth noting that most reports have stressed on complete surgical resection as the appropriate management of retroperitoneal schwannomas $[3,4]$. Our view is that the morbidity associated with surgical resection is not justified in these benign lesions, and the use of EUS-FNA to establish the diagnosis may help in avoiding unnecessary surgery.

\section{Competing interests: None}

Endoscopy_UCTN_Code_CCL_1AF_2AG
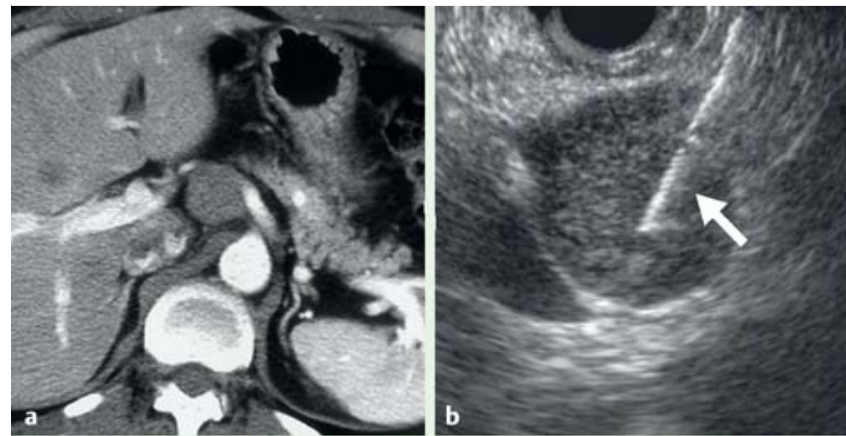

Fig. 1 Imaging findings and histopathological features. a Contrast enhanced computed tomography (CT) scan showing well-demarcated low density mass in the retroperitoneal region. b Endoscopic ultrasound image showing a fine needle inserted into the mass (arrow). c Spindle cells
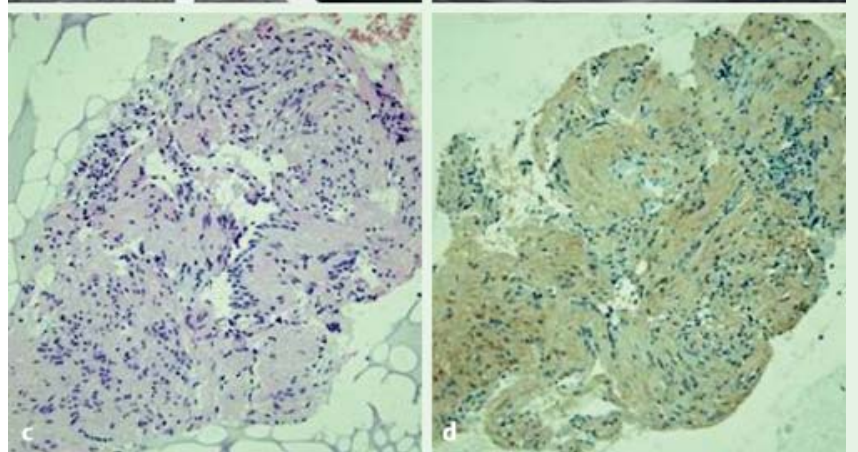
on cellblock sections, without any mitosis (hematoxylin and eosin stain, original magnification $\times 400$ ). d Immunohistochemical S-100 positive staining (magnification $\times 400)$.

S. Hijioka ${ }^{1}$, A. Sawaki ${ }^{1}$, N. Mizuno ${ }^{1}$, K. Hara ${ }^{1}$, M. A. Mekky², V. Bhatia ${ }^{3}$, W. Hosoda ${ }^{4}$, Y. Yatabe ${ }^{4}$, Y. Shimizu ${ }^{5}$, K. Tamada ${ }^{6}$, Y. Niwa ${ }^{7}$, K. Yamao ${ }^{1}$

1 Department of Gastroenterology, Aichi Cancer Center Hospital, Nagoya, Japan

2 Department of Tropical Medicine and Gastroenterology, Assiut University Hospital, Assiut, Egypt

3 Department of Medical Hepatology, Institute of Liver and Biliary Sciences, Delhi, India

4 Department of Genetic Pathology and Molecular Diagnostics, Aichi Cancer Center Hospital, Nagoya, Japan

${ }^{5}$ Department of Gastroenterological Surgery, Aichi Cancer Center Hospital, Nagoya, Japan

6 Department of Gastroenterology, jichi Medical University, Tochigi, Japan 7 Department of Endoscopy, Aichi Cancer Center Hospital, Nagoya, Japan

\section{References}

1 Okada N, Hirooka Y, Itoh A et al. Retroperitoneal neurilemoma diagnosed by EUS-guided FNA. Gastrointest Endosc 2003; 57: $790-792$

2 Facciorusso D, Federici T, Giacobbe A et al. Retroperitoneal neurilemoma diagnosed by endosonographically guided fine needle aspiration. J Clin Ultrasound 2006; 34: 241 243

3 Li Q Gao C, Juzi JT, Hao X. Analysis of 82 cases of retroperitoneal schwannoma. ANZ J Surg 2007; 77: 237-240

4 Goh BK, Tan YM, Chung YF et al. Retroperitoneal schwannoma. Am J Surg 2006; 192: $14-18$

Bibliography

Dol $10.1055 / \mathrm{s}-0030-1255786$

Endoscopy 2010; 42: E296

(c) Georg Thieme Verlag KG Stuttgart · New York ISSN 0013-726X

\section{Corresponding author \\ S. Hijioka}

Department of Gastroenterology

Aichi Cancer Center Hospital

1-1 Kanokoden

Chikusa-ku

Nagoya

Aichi 464-8681

Japan

Fax: +81-52-7642963

rizasusu@aichi-cc.jp 